\title{
Diffuse light and building history of the galaxy cluster Abell 2667
}

\author{
G. Covone ${ }^{1,2}$, C. Adami ${ }^{2}$, F. Durret ${ }^{3,4}$, J.-P. Kneib ${ }^{2}$, G. B. Lima Neto ${ }^{5}$, and E. Slezak ${ }^{6}$ \\ 1 INAF - Osservatorio Astronomico di Capodimonte, Naples, Italy \\ 2 Laboratoire d'Astrophysique de Marseille, Traverse du Siphon, 13012 Marseille, France \\ e-mail: giovanni . covone@oamp. fr \\ 3 Institut d'Astrophysique de Paris, CNRS, Université Pierre et Marie Curie, 98bis Bd Arago, 75014 Paris, France \\ 4 Observatoire de Paris, LERMA, 61 Av. de l'Observatoire, 75014 Paris, France \\ 5 Instituto de Astronomia, Geofísica e C. Atmosf./USP, R. do Matão 1226, 05508-090 São Paulo/SP, Brazil \\ ${ }^{6}$ Observatoire de la Côte d'Azur, BP 4229, 06304 Nice Cedex 4, France
}

Received 3 August 2005 / Accepted 8 May 2006

ABSTRACT

\begin{abstract}
Aims. We searched for diffuse intracluster light in the galaxy cluster Abell $2667(z=0.233)$ from HST images in three broad bandfilters.

Methods. We applied an iterative multi-scale wavelet analysis and reconstruction technique to these images, which allows to subtract stars and galaxies from the original images.

Results. We detect a zone of diffuse emission southwest of the cluster center (DS1) and a second faint object (ComDif) within DS1. Another diffuse source (DS2) may be detected at lower confidence level northeast of the center. These sources of diffuse light contribute to $10-15 \%$ of the total visible light in the cluster. Whether they are independent entities or part of the very elliptical external envelope of the central galaxy remains unclear. Deep VLT VIMOS integral field spectroscopy reveals a faint continuum at the positions of DS1 and ComDif but do not allow a redshift to be computed, so we conclude if these sources are part of the central galaxy or not. A hierarchical substructure detection method reveals the presence of several galaxy pairs and groups defining a similar direction to the one drawn by the DS1 - central galaxy - DS2 axis. The analysis of archive XMM-Newton and Chandra observations shows X-ray emission elongated in the same direction. The X-ray temperature map shows the presence of a cool core, a broad cool zone stretching from north to south, and hotter regions towards the northeast, southwest, and northwest. This might suggest shock fronts along these directions produced by infalling material, even if uncertainties remain quite large on the temperature determination far from the center.

Conclusions. These various data are consistent with a picture in which diffuse sources are concentrations of tidal debris and harassed matter expelled from infalling galaxies by tidal stripping and undergoing an accretion process onto the central cluster galaxy; as such, they are expected to be found along the main infall directions. Note, however, that the limited signal to noise of the various data and the apparent lack of large numbers of well-defined independent tidal tails, besides the one named ComDif, preclude definitive conclusions on this scenario.
\end{abstract}

Key words. galaxies: clusters: individual: Abell 2667 - X-rays: galaxies: clusters - galaxies: intergalactic medium

\section{Introduction}

The dense environment of galaxy clusters has a strong influence on galaxy evolution. Galaxies that enter galaxy clusters are affected by a large variety of physical processes during their interactions with the individual cluster galaxies, the intracluster medium (ICM), and the whole cluster gravitational field. However, the details and the relative role of these interactions at different epochs and in different cluster regions are not yet fully understood (see, e.g., Treu et al. 2003). Some of these processes are held responsible for removing gas from the disk of spiral galaxies (ram pressure stripping, see e.g. Gunn \& Gott 1972), the halo gas reservoir (tidal truncation of galactic halos; see, e.g., Natarajan et al. 2002), generally leading to a decreasing star formation rate (starvation, Larson et al. 1980).

Multiple high-speed encounters (harassment, Moore et al. 1998) are, on the other hand, responsible for removing stars from the disk of late-type galaxies and creating tidal debris. Numerical simulations (e.g., Calcáneo-Roldán et al. 2000; Napolitano et al. 2003; Sommer-Larsen et al. 2005) have shown that this might be the main mechanism leading to the formation of a population of intracluster stars bound not to individual galaxies but to the cluster as a whole. This population is now detected as a faint, diffuse optical source, the intracluster light (ICL). Moreover, such stellar population is also expected to be a relevant remnant of the merging events that lead to the formation of the central bright galaxy (e.g., Lin \& Mohr 2004). Therefore, the study of the ICL is expected to give important information on the physical processes transforming the galaxies accreted in dense enviroments and on the formation of the clusters brightest galaxies and their extended envelope.

The stellar population responsible for the ICL may be probed both by observations of the individual stars (see, for instance, the recent review in Arnaboldi 2004) and of the cumulative diffuse light. Deep imaging surveys are accumulating growing and detailed evidence of diffuse structures with various shapes in several clusters (e.g., Gregg \& West 1998; Feldmeier et al. 2002, 2003; Mihos 2003; Adami et al. 2005; Da Rocha \& Mendes de Oliveira 2005; Krick et al. 2006), employing a variety of techniques to face the challenging task of detecting a diffuse source with a surface brightness that can be as low as $\sim 1 \%$ of the night sky level. 
Another known difficulty is given by the connection between the ICL and the brightest cluster galaxy envelope (see for instance Uson et al. 1991), which has led to various definitions of ICL in literature. Therefore, some care is needed when comparing different estimates of the amount of light found in the ICL population and what is obtained from numerical simulations. In the following, we assume that the brightest cluster galaxy envelope has the same origin and nature as the population of stars bound to the cluster as a whole, and make no strict distinction between them.

Lin \& Mohr (2004) have studied the near-infrared properties of a sample of 93 nearby, X-ray selected clusters and groups, showing that ICL is a common feature, accounting for a large part of the present total amount of stars (up to $50 \%$ for $\geq 10^{15} M_{\odot}$ clusters). The ICL contribution appears to increase with the total cluster mass. Note that in this work no distinction has been made between the ICL and the brightest cluster galaxy envelope.

In a sample of 24 clusters at $z<0.13$, Gonzalez et al. (2005) have used a two-component $r^{1 / 4}$ profile to fit the central elliptical galaxy and the population of intracluster stars. The outer component is found to be distributed on scales $\sim 10-40$ that are times larger than the inner one and $\sim 10$ times more luminous. Moving at higher redshifts, Zibetti et al. (2005) show evidence of the presence of ICL in rich galaxy clusters in the redshift range $0.2-0.3$. They stacked a sample of 683 galaxy clusters from the Sloan Digital Sky Survey and find that the ICL contribution to the total cluster light in the stacked cluster is $10.9 \pm 5.0 \%$, that it shares similar colors with the cluster population (within the photometric errors), but has a higher central concentration.

Numerical simulations have also greatly improved the description of the behavior of the diffuse light component. For instance, by using a high-resolution collisionless simulation, Napolitano et al. (2003) have found that in a Virgo-like cluster the velocity distribution of the ICL stars is strongly nonGaussian also at $z=0$, constituted of a wide spectrum of structures (filaments, concentrations of particles, etc.) and showing a clearly unrelaxed state, with a typical clustering radius of about $50 \mathrm{kpc}$. Murante et al. (2004) confirm with numerical simulations that the ICL contribution to the stellar mass seems to increase with the cluster mass and represents more than $10 \%$ of the total amount of stars. They also find the ICL stars to be older than galactic stars. Sommer-Larsen et al. (2005) find an intracluster star contribution to the total cluster light in the $B$ band of 20 to $40 \%$ at $z=0$, with a $B-R$ color of $1.4-1.5$ and a metallicity varying from solar to half solar.

The number of clusters with individual well studied diffuse light sources is, however, still quite small and largely limited to the local Universe. In order to test predictions from the numerical simulations at different cosmological epochs, it is therefore mandatory to enlarge this limited sample.

In this framework, we studied the moderately distant galaxy cluster, Abell $2667(z=0.233)$. Abell 2667 is a known gravitational-lensing cluster with several multiple image systems, including a bright giant gravitational arc with the source located at $z=1.0334$ (Covone et al. 2006a). This is a massive $\mathrm{X}$-ray luminous galaxy cluster: within the range $[0.1-2.4] \mathrm{keV}$, its X-ray luminosity is estimated to be $L_{X}=(14.90 \pm 0.56) \times$ $10^{44} h_{70}^{-2} \mathrm{erg} \mathrm{s}^{-1}$ (Rizza et al. 1998). Its X-ray morphology (Rizza et al. 1998) and galaxy dynamical analysis (Covone et al. 2006a) support the idea that its inner core is relaxed, while wide field imaging shows that the cluster is still accreting galaxies and galaxy groups (H. Ebeling, private communication). Recently, Covone et al. (2006a) performed a spectroscopic survey of the cluster core by using the VIMOS Integral Field Unit (IFU)
Table 1. Properties of the WFPC2 data (AB system).

\begin{tabular}{lccc}
\hline \hline Filter & $\begin{array}{c}\text { Exp time } \\
\mathrm{s}\end{array}$ & $\begin{array}{c}\text { Zero point } \\
\mathrm{mag}\end{array}$ & $\begin{array}{c}\text { Sky level } \\
\mathrm{mag} / \operatorname{arcsec}^{2}\end{array}$ \\
\hline$F 450 W$ & 12000 & 21.94 & 23.44 \\
$F 606 W$ & 4000 & 22.99 & 23.09 \\
$F 814 W$ & 4000 & 22.08 & 22.39 \\
\hline
\end{tabular}

and compared the mass models obtained through gravitational lensing, cluster galaxy dynamics, and X-ray gas temperature. The spectroscopically confirmed galaxy population in the cluster core is made up of evolved galaxies with no detectable sign (in the optical bands) of current or very recent star formation. The brightest cluster galaxy shows a bright region with emission lines ([OII], $\mathrm{H} \beta$, [OIII], $\mathrm{H} \alpha$ ) extended along the NE-SW direction and is thought to be associated with a cooling flow (Rizza et al. 1998; see also Covone 2004).

Our aims are (i) to probe diffuse light physics in an earlier stage than most of the existing works, as Abell 2667 is located at a redshift of 0.233 , and (ii) to use the detected ICL sources to describe and discuss the building history of this cluster by means of a new dynamical analysis of the cluster galaxies (based on the Covone et al. 2006a redshift catalog) and of archive X-ray data.

The paper is organized as follows: the optical data and analysis methods are presented in Sect. 2. Imaging and spectroscopic results on the ICL are described in Sect. 3. The X-ray observations and results are presented in Sect. 4, and a possible building scenario for Abell 2667 is discussed in Sect. 5. Conclusions are drawn in Sect. 6. Throughout this paper we assume a cosmological model with $\Omega_{\mathrm{m}}=0.3, \Omega_{\Lambda}=0.7$, and $H_{0}=70 \mathrm{~km} \mathrm{~s}^{-1} \mathrm{Mpc}^{-1}$. At $z=0.233$ the angular scale is thus $3.722 \mathrm{kpc} / \operatorname{arcsec}$. Magnitudes are given in the AB system.

\section{Optical data and analysis}

The imaging and spectroscopic data used in this work have already been presented in Covone et al. (2006a). We refer to that paper for details on the data reduction. In this section we present only the most relevant properties of the dataset, the image analysis and the hierarchical analysis of the spectroscopic catalog.

\subsection{The optical data set}

Detection of diffuse light was performed on archive Hubble Space Telescope (HST) WFPC2 images. These are three broad band filters: $F 450 W$ (exposure time 12000 s), F606W (4000 s), and $F 814 W(4000$ s). Using the IRAF package drizzle, we obtained a pixel scale of 0.05 arcsec per pixel. The photometric properties of the given multiband dataset are summarized in Table 1.

An image of the central region of Abell 2667 is displayed in Fig. 1, together with the VIMOS-IFU field-of-view (f.o.v.), which covers a region of about $220 \times 220 \mathrm{kpc}^{2}$ around the central galaxy.

Integral field spectroscopy was obtained with the VIMOSIFU mounted on the VLT Melipal, as part of an integral field spectroscopic survey of X-ray bright clusters at $z \simeq 0.2$ showing strong gravitational lensing features (Covone et al. 2006b). Observations were performed using the low-resolution blue grism with a fiber diameter of 0.66 arcsec. The useful wavelength range is between $\sim 3900 \AA$ and $6800 \AA$ with spectral resolution $R \simeq 250$. The total exposure time was $10800 \mathrm{~s}$, divided into four exposures with a $\sim 1$ arcsec offset among them. 


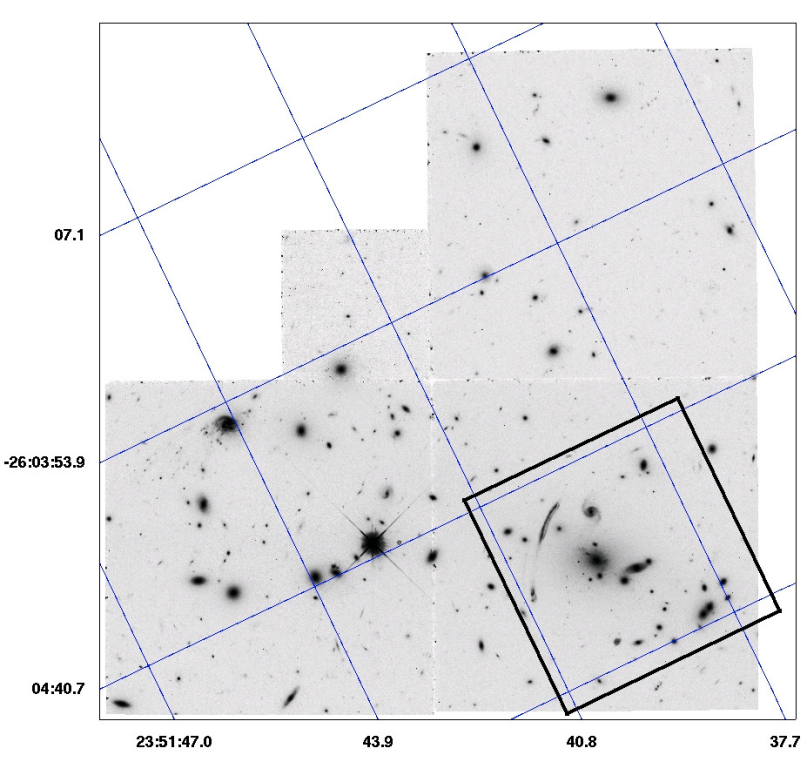

Fig. 1. WFPC2 image of Abell 2667 in the $F 606 \mathrm{~W}$ filter. The thick box indicates the VIMOS-IFU field of view $\left(54 \times 54 \operatorname{arcsec}^{2}\right)$. The overlayed grid shows the North orientation towards the upper left.

\subsection{Image analysis}

To detect large scale faint diffuse light emission sources in the presence of structures (i.e., stars and galaxies), we first need to remove these small-scale objects. To obtain an image containing only these objects, we performed a multi-scale analysis of the images, assuming that the spatial extension of the diffuse sources is in most cases larger than the typical galaxy scale. Diffuse light is searched for after subtraction of all these smaller scale sources. We only analyzed the area covered by the WFPC2-wf3 chip, since this encloses the cluster central region and the VIMOS-IFU f.o.v. We summarize our method here and refer to Adami et al. (2005) for a full description. Recently, Da Rocha \& Mendes de Oliveira (2005) applied a similar technique to search for diffuse light in compact groups of galaxies.

This method involves a thresholding of the data in the wavelet space in order to remove the noise locally without smoothing the astrophysical signal. Wavelet coefficients are considered to be significant on a given scale when their absolute magnitude exceeds three times the rms fluctuation expected for the wavelet coefficients of white noise on the same scale. The image analysis was performed twice. From the first subset of significant wavelet coefficients, a positive image in real space was restored. This image was subtracted from the original one and the wavelet transform of the residual was thresholded with thresholds similar to those in the first iteration. Applying the restoration algorithm to the second subset of coefficients enables one to obtain an image that includes the previously hidden significant features.

In this way, adding together the two restored images with a constraint for the result to be positive (cf. the subtraction step) enables one to obtain a more adequate map of the small-scale structures within the image. This last map can then be subtracted from the original image, leading to a new residual image where large-scale sources can be quite easily searched for. Hence, the final products of this process are an image of what we call the objects, i.e. the signal with a characteristic scale smaller than the maximal scale used, and a residual image exhibiting mostly features with a characteristic scale larger than this maximum

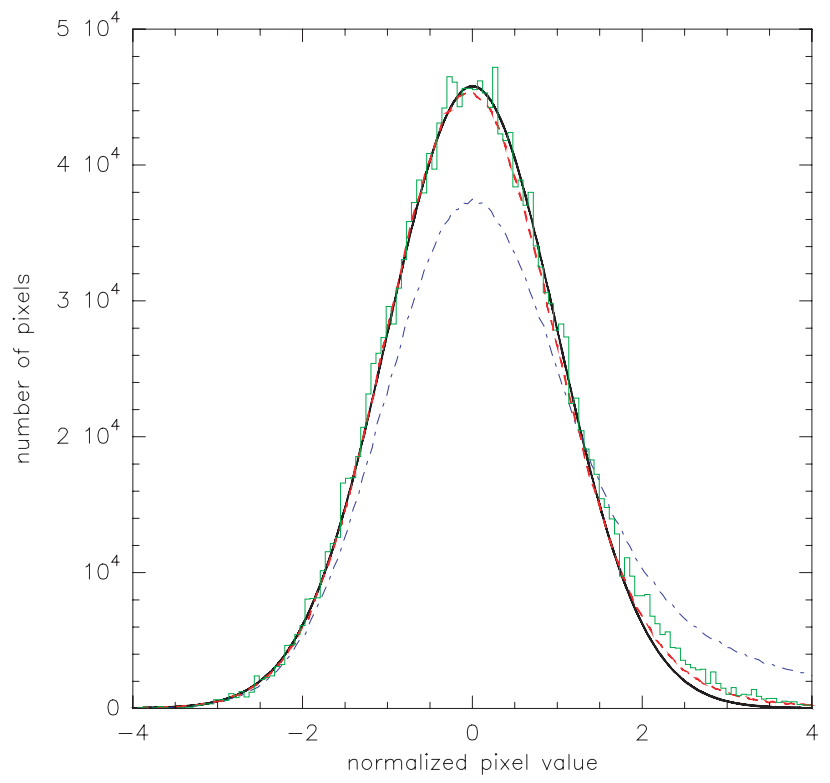

Fig. 2. Comparison between the distibution of pixel values before and after the removal of small-scale features in the WFPC2 F814W image. The pixel values are normalized with respect to the rms fluctuation measured in the residual image. The blue dot-dashed line and the red dashed line show the distributions of pixel values for the initial and the residual images, respectively. The black solid line stands for a Gaussian fit of the pixel distribution in the residual image. The green histogram shows the distribution of pixel values in the residual image for an empty background region (with size $200 \times 200$ pixels).

value. This value is $\sim 7 \operatorname{arcsec}$ for the first iteration and $\sim 2 \operatorname{arcsec}$ for the second iteration.

We checked that the noise in the residual image has nearly Gaussian statistical properties, as shown in Fig. 2. The difference between the pixel histogram and the Gaussian fit slightly decreases the statistical significance of the detected features (a $3 \sigma$ level is, e.g., translated into a $2.5 \sigma$ level). First, the applied procedure succeeds in removing the tail of the distribution related to bright pixels linked to objects (i.e., stars and galaxies). Second, the histogram of pixel values in the residual image is nearly Gaussian. The pixel value distribution of the non-drizzled residual image shows the same behavior. Therefore, the correlation between neighboring pixels introduced by drizzling has a very small effect and we neglected it when computing the variance of the noise. Note that the statistical significance of any feature detected in the residual image has been estimated with respect to the Gaussian fit.

A few elongated objects that appear to be possible tidal debris within the cluster are not detected as diffuse sources by the present approach because they have characteristic sizes smaller than 2 arcsec. Finally, we checked that known strong gravitational lensing features are not misclassified as diffuse sources or tidal debris, by verifying that they are all included in the object map in all filters. The results of the described multi-scale method are described in Sect. 3.

\subsection{Serna \& Gerbal (1996) hierarchical analysis}

The redshift catalog is presented in Covone et al. (2006a). This is a complete catalog down to total magnitudes of $V_{606}=$ 21.5 over the central $\sim 1 \operatorname{arcmin}^{2}$. We selected galaxies between $z=0.220$ and $z=0.244$ in order to remove obvious interlopers from the redshift histogram, and applied the 


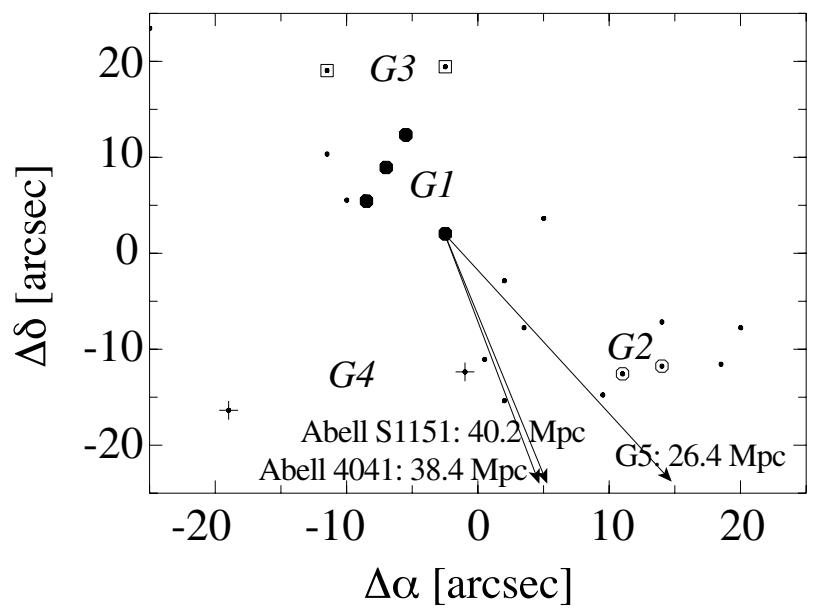

Fig. 3. Location of the subgroups detected within Abell 2667. Dots: positions of galaxies with redshifts between $z=0.22$ and $z=0.244$ in the Abell 2667 IFU field of view (in arcsec relative to the geometrical center of the galaxies measured spectroscopically). The galaxies forming the central group according to the Serna \& Gerbal method are indicated with filled black circles. The open circles show two galaxies forming the G2 pair towards the SW of the cluster. Squares are for the G3 pair and stars for the minor G4 pair. Lines indicate the directions of major nearby structures (Abell 4041, Abell S1151 and G5). North is up and East to the left.

Serna \& Gerbal (1996, hereafter SG) hierarchical method to this sample with the aim of detecting substructures within the cluster core.

This method calculates the relative binding energies of all the galaxies on the basis of the measured redshifts and magnitudes. We used a mass-to-light $(M / L)$ ratio in the $B$ band of 450 (see, e.g., Łokas \& Mamon 2003). We checked that using $M / L$ between 320 and 580 (i.e., large enough to encompass most of the $M / L$ ratios in galaxy clusters) does not change the results, both qualitatively and quantitatively.

We identify four groups of galaxies:

- a first group of four galaxies (hereafter, G1) in the central region, which has the largest (in absolute value) binding energy within the selected sample;

- a pair of galaxies (G2) SW of G1 with a binding energy $\simeq 6$ times lower than that of $\mathrm{G} 1$;

- two minor galaxy pairs north of G1 (hereafter, G3) and south of G1 (hereafter, G4), with respective binding energies 40 and 1200 times smaller than that of G1.

In view of these numbers, we consider in the following only the groups G1, G2, and G3. The positions of the galaxies in these structures are shown in Fig. 3. Both G2 and G3 have mean redshifts of $z=0.2331$ and 0.2262 , respectively, therefore they are galaxy pairs within Abell 2667.

\section{Diffuse light in Abell 2667}

In the following section we describe the photometric and spectroscopic properties of the diffuse sources detected in the cluster.

\subsection{Imaging of the diffuse sources}

Using the multi-scale approach described in Sect. 2, we identified two distinct diffuse sources in the central $200 \times 200 \mathrm{kpc}^{2}$. The brightest source (DS1, hereafter) is located about 20 arcsec
SW of the cluster center, while the second one (DS2, hereafter) is about 25 arcsec from the cluster center in the NE direction. As shown in Fig. 4, diffuse emissions appear at the same positions and with roughly the same extensions in the three residual images.

In order to estimate the statistical significance of the detections of the two diffuse sources, we estimated the mean and standard deviation of the images in the three bands in an external area where no diffuse source was present. We compared this value to the mean level of the two diffuse sources. This allowed us to estimate the values of the detection significances given in the last columns of Table 2.

The DS1 source is detected in the residual images of the two redder filters at respective peak significance levels of $1.9 \sigma$ and $2.7 \sigma$ in the $F 606 \mathrm{~W}$ and $F 814 \mathrm{~W}$ filters, respectively. Its average surface brightness is $V_{606} \simeq 26.2 \mathrm{mag} \mathrm{arcsec}{ }^{-2}$. There is also a possible detection for DS1 in the $F 450 \mathrm{~W}$ filter but with a lower significance $(\sim 1 \sigma)$; this detection corresponds to a total magnitude of $B_{450}=21.9$.

The DS2 source is detected with lower significance levels of $1.5 \sigma$ and $2.1 \sigma$ in the $F 606 \mathrm{~W}$ and $F 814 \mathrm{~W}$ images, respectively. Its average surface brightness is $V_{606} \simeq 26.7 \mathrm{mag} \mathrm{arcsec}^{-2}$. Table 2 summarizes the photometric properties of these two diffuse sources: the total magnitudes, the extension (in the $F 814 \mathrm{~W}$ filter), the average surface brightness (mag/arcsec ${ }^{2}$, and detection levels in the three given filters. Total magnitudes in the three bands were computed in the areas where the sources were detected in the $F 814 W$ filter.

A small compact and well-defined object is present inside the diffuse source DS1 and is excluded from the rest of our analysis. A second very faint object is also observed within DS1 (see Fig. 5) and is called the "compact diffuse source" (ComDif). Photometry and morphological characteristics for this object have been computed using SExtractor.

We compared the colors of the diffuse sources to those of the spectroscopically confirmed cluster galaxies and those of late-type stars (from the Pickles 1998 library) redshifted at $z=0.233$ (see Fig. 6). The two sources have similar colors within the present photometric uncertainities and close to those of evolved and K-type giants, as already found for the tidal debris in Centaurus A by Calcáneo-Roldán et al. (2000). They appear to have redder average $V-I$ colors than the evolved cluster population and bluer average $B-V$ colors.

When compared to the colors of the redshifted spectral templates of galaxies from the Kinney-Calzetti spectral atlas (Kinney et al. 1996) - but without taking evlution into account - we find that their $B-V$ color is between that of an Sbc galaxy and an S0 galaxy.

\subsection{Spectra of DS1 and ComDif}

Using the spatial information for the detected diffuse sources, we extracted their spectra from the VIMOS-IFU data cube (Covone et al. 2006a), avoiding the known compact sources in that area (Fig. 7). In total, we used 33 and 267 fibers (i.e., about 11 and $91 \operatorname{arcsec}^{2}$ ) for ComDif and DS1, respectively. We could not obtain a reliable spectrum for the lower surface brightness source DS2, as it was located in a region covered by a set of dead fibers. We plot the spatially averaged, one-dimensional spectra of the DS1 and ComDif regions in Fig. 8, together with a local sky spectrum (i.e., the summed spectrum from nearby fibers in the same quadrant, not covering any detected object).

The detection of the spectrum from faint and extended sources in a VIMOS-IFU data-cube is a demanding task. 


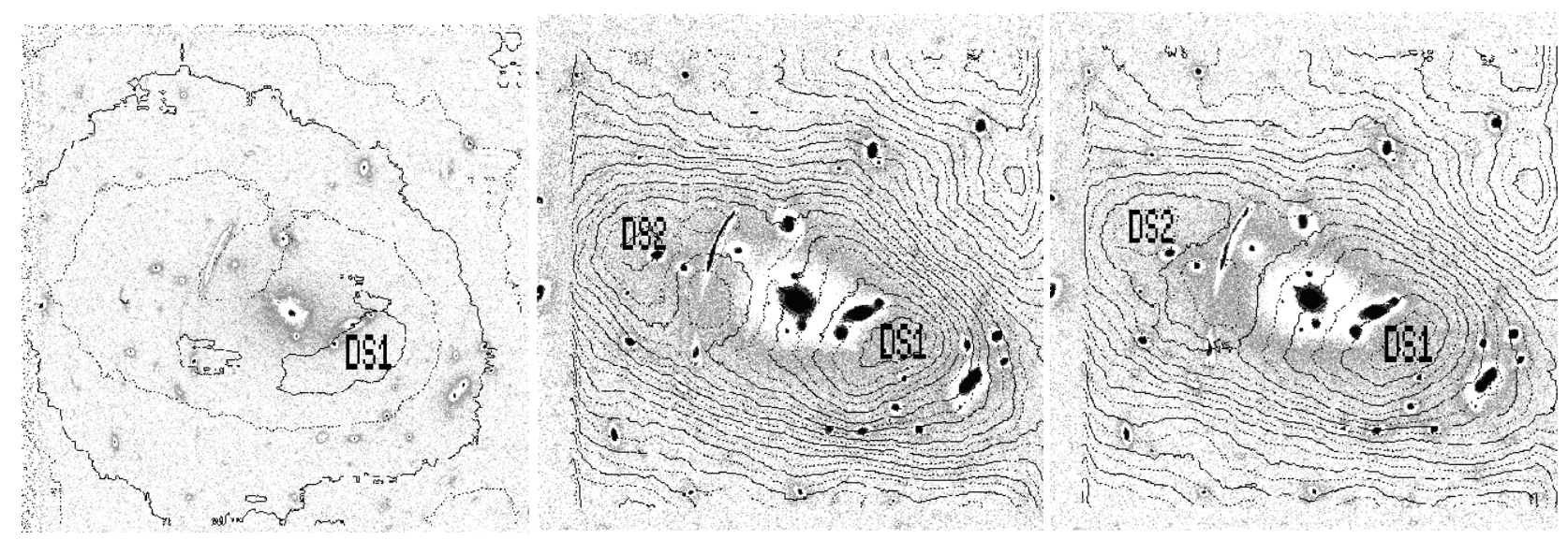

Fig. 4. Residual WFPC2 images in the $F 450 W$ (left), F606W (middle) and $F 814 W$ (right) bands. Each figure is $1.2 \times 1.2$ arcmin $^{2}$. North is up and East to the left.

Table 2. Properties of the ICL sources detected in Abell 2667.

\begin{tabular}{lccccccccccc}
\hline \hline Source & $\begin{array}{c}\alpha \\
(\text { degree })\end{array}$ & $\begin{array}{c}\delta \\
(\text { degree })\end{array}$ & $B_{450}$ & $V_{606}$ & $I_{814}$ & $\begin{array}{c}\text { area } \\
\left(\operatorname{arcsec}^{2}\right)\end{array}$ & $\mu_{450}$ & $\mu_{606}$ & $\mu_{814}$ & $F 606 W$ & $F 814 W$ \\
\hline Diffuse sources & & & & & & & & & & \\
DS1 & 357.910 & -26.088 & 21.9 & 20.9 & 19.9 & 137 & 27.2 & 26.2 & 25.2 & $1.9 \sigma$ & $2.7 \sigma$ \\
DS2 & 357.920 & -26.079 & - & 21.3 & 20.6 & 143 & - & 26.7 & 26.0 & $1.5 \sigma$ & $2.1 \sigma$ \\
Compact diffuse source & & & & & & & & & & & - \\
ComDif & 357.9096 & -26.0880 & 27.29 & 25.77 & 24.12 & 2.4 & 28.24 & 26.72 & 25.07 & - & - \\
\hline
\end{tabular}

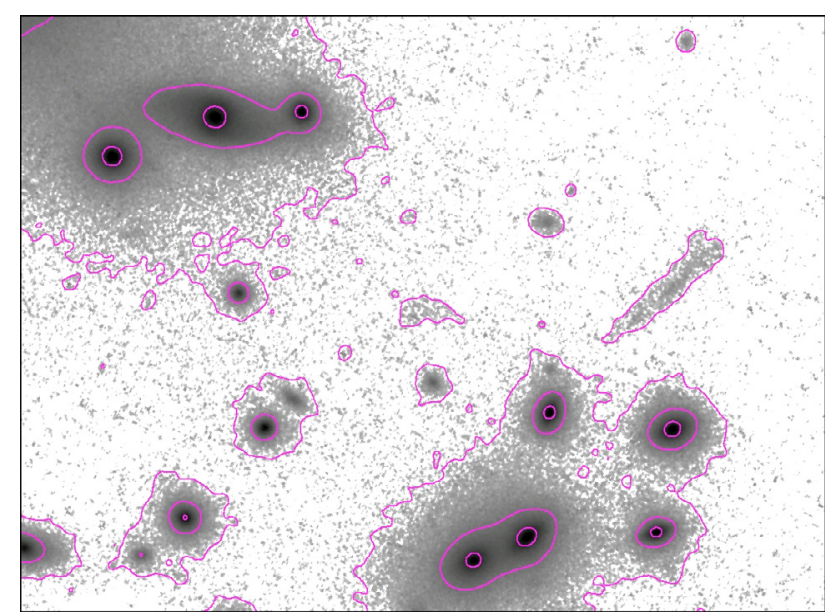

Fig. 5. Zoomed image ( $F 814 W$ filter) of the region around the ComDif diffuse source (at the center of the image). Contour levels correspond to surface brightness levels of $I_{814}=23.0,22.0,21.0 \mathrm{mag} / \operatorname{arcsec}^{2}$. The field is $0.5 \times 0.3 \operatorname{arcmin}^{2}$ wide. North is up and East to the left. The elongated object $\sim 10 \operatorname{arcsec} \mathrm{W}$ of the center is a background lensed object ( $\mathrm{Rc}$ in Covone et al. (2004); photometric redshift $z=1.15$ ).

The large extension of the source makes the removal of the background signal very difficult. In this situation the statistical approach generally used to remove the background signal in the VIMOS-IFU (Zanichelli et al. 2005), i.e. computing the sky in each module of 400 fibers, is clearly not appropriate, since it would remove any signal from extended sources that may cover a large fraction of the considered fibers. Therefore, we produced a data-cube with no such background removal and evaluated the sky in regions with no detected objects close to the considered sources. To take into account the fact that fibers have different transmissions, fiber spectra have been renormalized using the

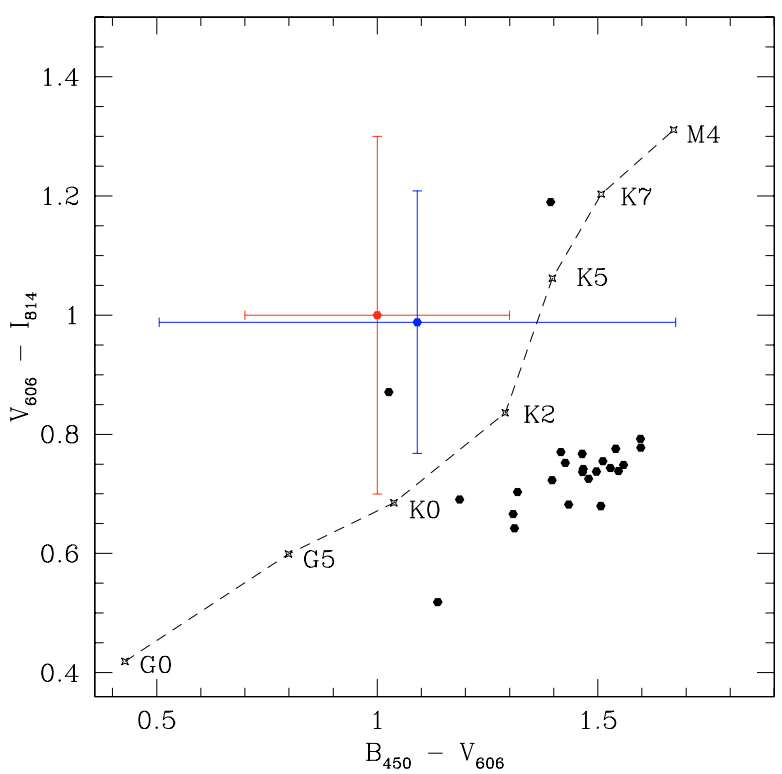

Fig. 6. Color-color diagram for the spectroscopically confirmed cluster galaxies (filled circles), the DS1 (red symbol with smaller error bar along the $B-V$ axis), and ComDif (blue symbol with smaller error bars along the $V-I$ axis) sources and the Pickles (1998) late-type star templates redshifted at $z=0.23$ (crosses connected by a line).

flux measured in a given sky line, as described in Zanichelli et al. (2005; see also Covone et al. 2006b). However, as the background signal in the VIMOS-IFU is a strong function of position, this approach introduces further noise in the extracted spectra. Unfortunately, this is particularly true in the spectral range $\lambda \sim 4800-4900 \AA$ where the signal from the CaII H and $\mathrm{K}$ absorption lines from objects at rest in the cluster potential well is expected. 


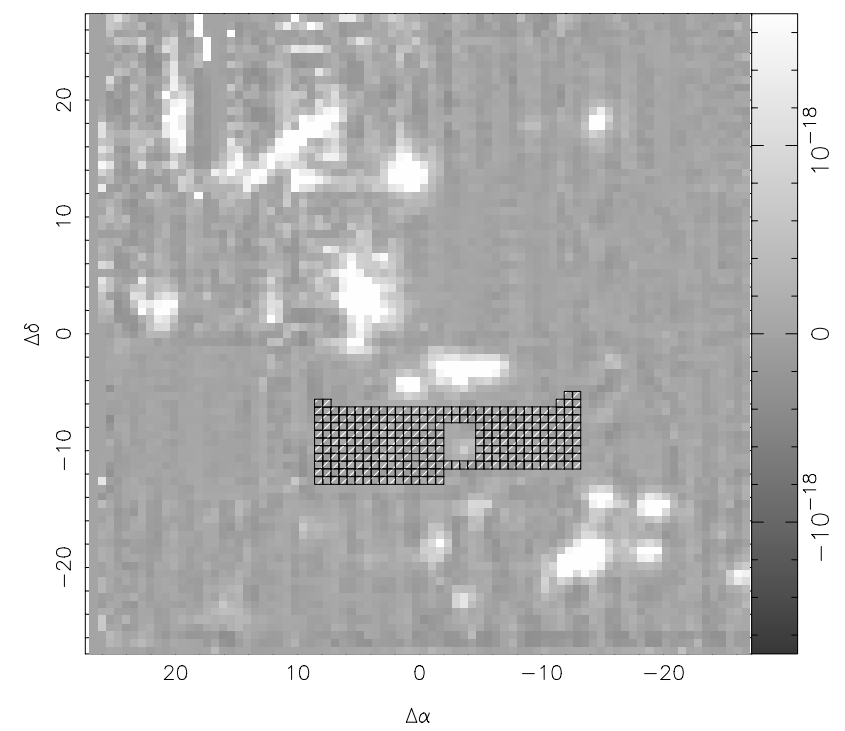

Fig. 7. Positions of the fibers of the VIMOS-IFU considered to obtain the spectrum of the diffuse emission discovered SW of the cluster (DS1). The hole in the grid corresponds to an obvious object that was taken out. North is up and East to the left.

As shown in Fig. 8, we clearly detect the continuum of both ComDif and DS1, thus supporting the photometric detection since no such continuum is detected in areas without visible ICL. However, the low $\mathrm{S} / \mathrm{N}$ ratio and the noise spikes due to the spatially varying background prevent us from detecting any significant absorption feature. The redshift of ComDif would be $0.235 \pm 0.003$ if we assume the H\&K lines as real. Deeper data would be needed to confirm these results.

We note that no emission line is detected at the given positions. We can set an upper limit $\left(f_{1} \simeq 0.7 \times 10^{-18} \mathrm{erg} \mathrm{s}^{-1} \mathrm{~cm}^{-2}\right)$ to any emission line (covering a spatial extension of one fiber). Such an emission line absence most probably implies that DS1 is made up of stars and not of ionized gas.

\section{X-ray analysis}

We used both Chandra and XMM-Newton archival data, and taken advantage of the Chandra high-resolution imaging to search for fine morphological structures in the gas distribution and of the XMM-Newton high sensitivity to obtain spatially resolved X-ray spectra.

\subsection{Chandra data}

The Chandra observation was performed in June 2001 with the ACIS-S detector (observation ID 2214, P.I. S. Allen). The data was taken in Very Faint mode with a time resolution of $3.24 \mathrm{~s}$, during $11 \mathrm{ks}$. The CCD temperature was $-120^{\circ} \mathrm{C}$.

We re-ran the "pipeline", following the standard data reduction procedure, Standard Data Processing, producing new level 1 and 2 event files with CIAO version 3.2 and CALDB version $3.0 .0^{1}$. We restricted our data reduction and analysis to the back-illuminated chip, ACIS-S3 (the cluster is completely contained in this CCD chip).

We used the "blank-sky" CTI-corrected ACIS background event files, made available by the ACIS calibration team ${ }^{2}$. The

\footnotetext{
${ }^{1}$ http://asc.harvard.edu/ciao/

${ }^{2}$ http://cxc.harvard.edu/cal/Acis/WWWacis_cal.html
}
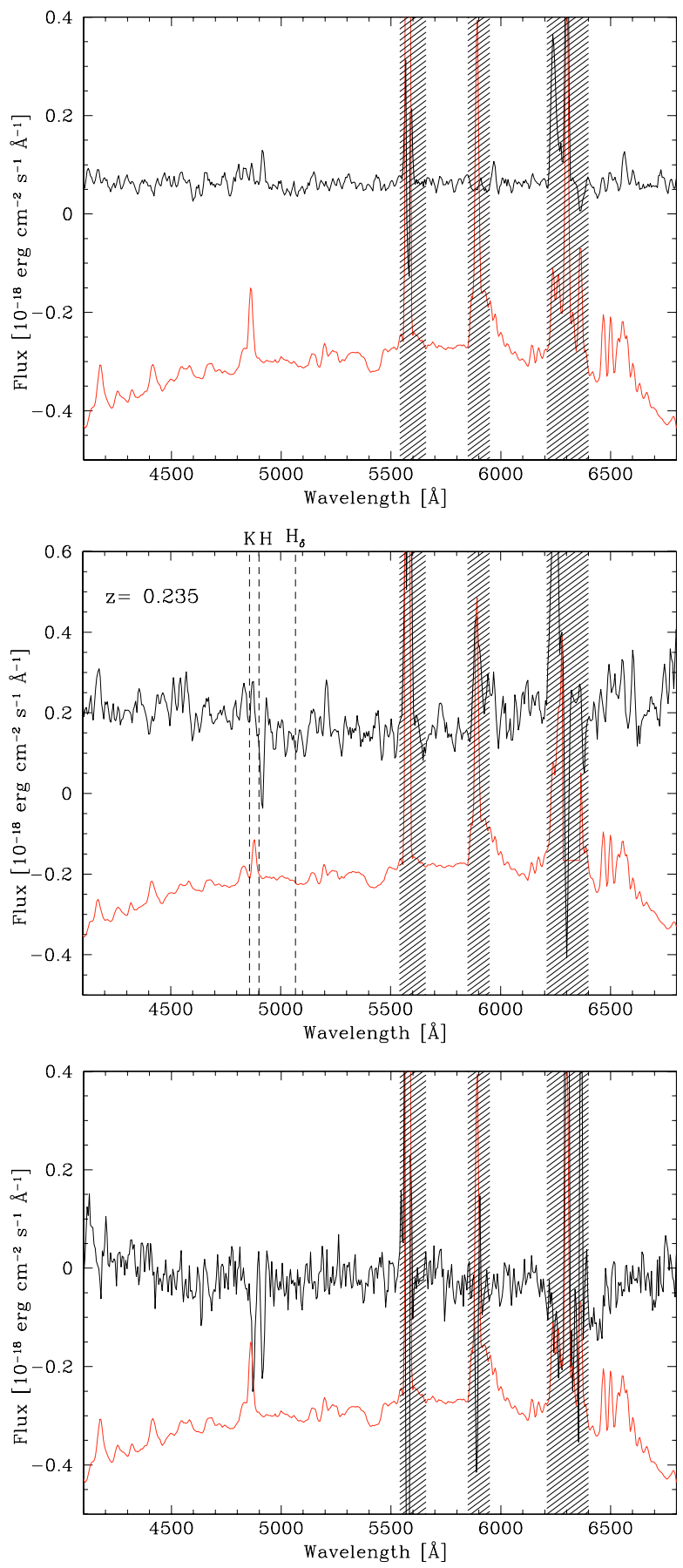

Fig. 8. Spectra of the entire DS1 diffuse source (upper panel) of the compact diffuse source ComDif (middle panel) and of an empty region nearby (lower panel), together with a scaled background signal at the same locations (in red). Spectra have been Gaussian smoothed with a $F W H M=15 \AA$. The expected positions of some absorption lines at redshift $z=0.235$ are shown (see text for discussion). Shaded regions mark the spectral ranges where sky signal is dominant.

background events were filtered, keeping the same grades as the source events, and then were reprojected to match the sky coordinates of the Abell 2667 ACIS observation. We restricted our analysis to the range $[0.3-8.0 \mathrm{keV}]$, because above $8.0 \mathrm{keV}$ the $\mathrm{X}$-ray emission is largely background-dominated. 


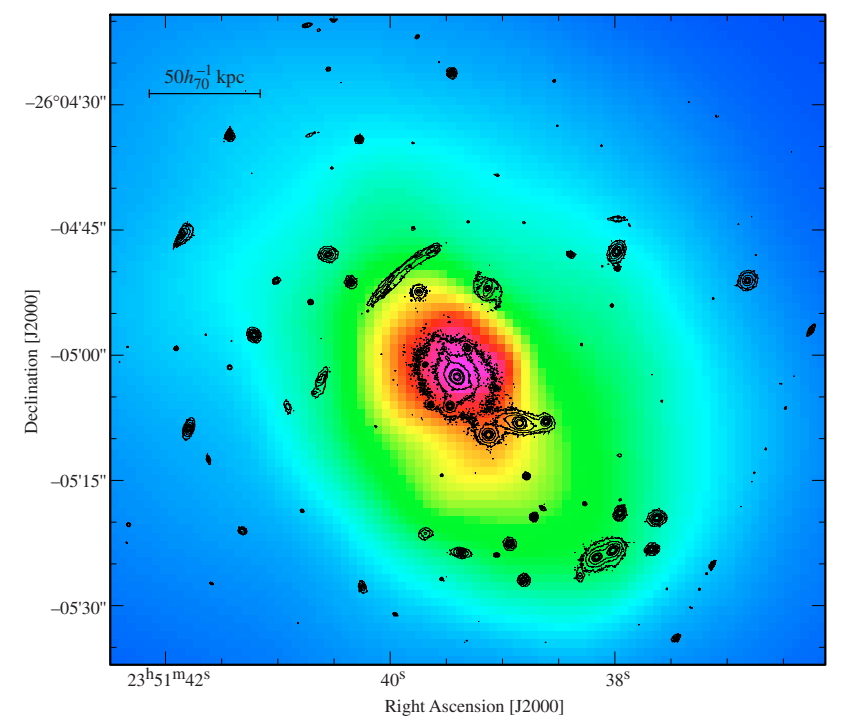

Fig. 9. Chandra ACIS-S3 X-ray adaptively smoothed image of Abell 2667 in the [0.3-8.0 keV] band, corrected by the exposure map and binned by a factor of $4(1$ pixel $=2$ arcsec $)$. The HST/WFPC 2 contours (filter $F 814 W$ ) are shown as black lines.

\subsection{XMM-Newton data}

Abell 2667 was observed in June 2003 (revolution 647, exposure 0148990101, P.I. S. Allen) during 26 ks, in standard Full Frame mode using the "medium" filter with the EPIC MOS1, MOS2, and $\mathrm{PN}$ detectors. We used the standard procedure and produced "clean" event files. The light-curves in the [10-12 keV] band showed that there were flaring events during the observation. Filtering out the periods with flares reduced the exposure times to $22226 \mathrm{~s}, 22502 \mathrm{~s}$, and $15092 \mathrm{~s}$ for MOS1, MOS2, and PN, respectively.

With the cleaned and filtered event files, we created the redistribution matrix file (RMF) and ancillary response file (ARF) with the XMM-Newton Science Analysis System (SAS) tasks rmfgen and arfgen for each camera and for each region that we analyzed. The background was taken into account by extracting spectra from the blank sky templates described by Lumb et al. (2002), and reprojected to the coordinates and roll angle of Abell 2667.

\subsection{Chandra $X$-ray imaging}

We constructed an adaptively smoothed image in the [0.3-8.0 keV] band using the CSMOOTH tool from CIAO. The ACIS-S3 point spread function (PSF) has an on-axis spatial $F W H M \approx 0.8$ arcsec, weakly dependent on the energy, and the unbinned pixel size is $0.5 \operatorname{arcsec}^{3}$. The exposure map was generated by the script MERGE_ALL, where we calculated the spectral weights needed for the instrument map, using the cluster total spectrum, i.e., the spectrum obtained inside a circle of radius of 1 arcmin centered on the cluster center.

Figure 9 shows the Chandra ACIS-S3 image with HST isocontours superimposed. The offset between the center of the main X-ray emission and the central galaxy is only about 1.0 arcsec (i.e., less than $4 h_{70}^{-1} \mathrm{kpc}$ ). The overall X-ray emission is elliptical and aligned with the galaxy distribution. A secondary $\mathrm{X}$-ray peak is observed about $10 \operatorname{arcsec} \mathrm{SW}$ of the cluster center

\footnotetext{
${ }^{3}$ http://cxc.harvard.edu/cal/Acis/Cal_prods/psf/ psf.html
}

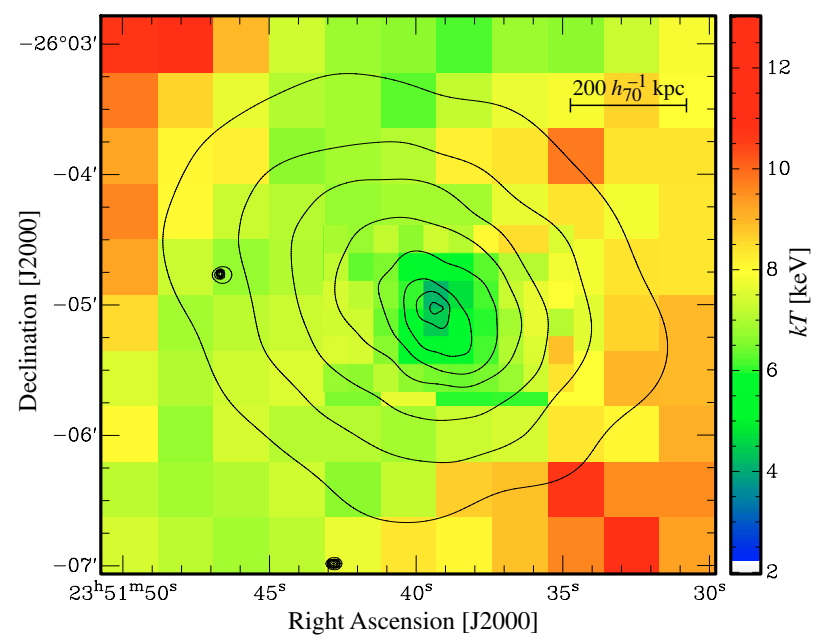

Fig. 10. XMM temperature map of the X-ray gas in the central region. The contours correspond to the Chandra surface brightness as in Fig. 9.

at the position of a bright galaxy. Farther along the same direction, $\simeq 29$ arcsec away from the center, there is a third, smaller $\mathrm{X}$-ray peak coinciding with a galaxy pair.

\subsection{X-ray spectral analysis and temperature map}

The EPIC-MOS and PN PSF have an FWHM $\approx 5$ arcsec, but their PSF have somewhat extended wings and the half energy width is $\approx 14 \operatorname{arcsec}^{4}$ The spectral analysis is not limited by the spatial resolution but rather by the binning necessary to achieve the sufficient $\mathrm{S} / \mathrm{N}$ ratio required for spectral fitting.

The temperature map was computed from XMM-Newton data following Durret et al. (2005). Briefly, the event files were rebinned with a pixel size of 25.6 arcsec and for each pixel in the grid we computed the RMF and ARF matrix and fit a MEKAL (Kaastra \& Mewe 1993; Liedahl et al. 1995) plasma model using XSPEC version 11.2. For the inner region only, a rectangle of $76.8 \times 128 \mathrm{arcsec}^{2}$, we used a higher resolution pixel of 12.8 arcsec. We set a minimum count number of 1200 counts per pixel. When we had enough counts, the spectral fit was done with the hydrogen column density fixed at the local Galactic value. For each pixel, we estimated $N_{\mathrm{H}}$ using the task nh from FTOOLS (which is an interpolation from the Dickey et al. 1990 Galactic $N_{\mathrm{H}}$ table).

Figure 10 shows the temperature map in the central region of Abell 2667. The gas is cooler in the central $R \approx 13 \operatorname{arcsec}$ with a temperature $k T=4.2 \pm 0.3 \mathrm{keV}$. The coolest pixel coincides with the main X-ray intensity peak. At the limits of the map, the temperature reaches about $12 \mathrm{keV}$. The ratio between the high and low temperatures is about 3 , as observed in most coolingcore clusters (e.g. Piffaretti et al. 2005).

In contrast to the surface brightness map, which shows elliptical symmetry, the temperature map shows a cooler lane, roughly stretching from north to south, with hotter regions towards NE, SW and, to a smaller level, NW relatively to the cluster center. The general direction of the cold lane is approximately perpendicular to the principal axis of the X-ray emission.

We drew the temperature profile by extracting spectra in concentric circles (see Fig. 11). This profile confirms that the temperature is notably lower in the innermost $150 \mathrm{kpc}$ of the

\footnotetext{
4 http://xmm.vilspa.esa.es/external/ xmm_user_support/documentation/uhb/index.html
} 


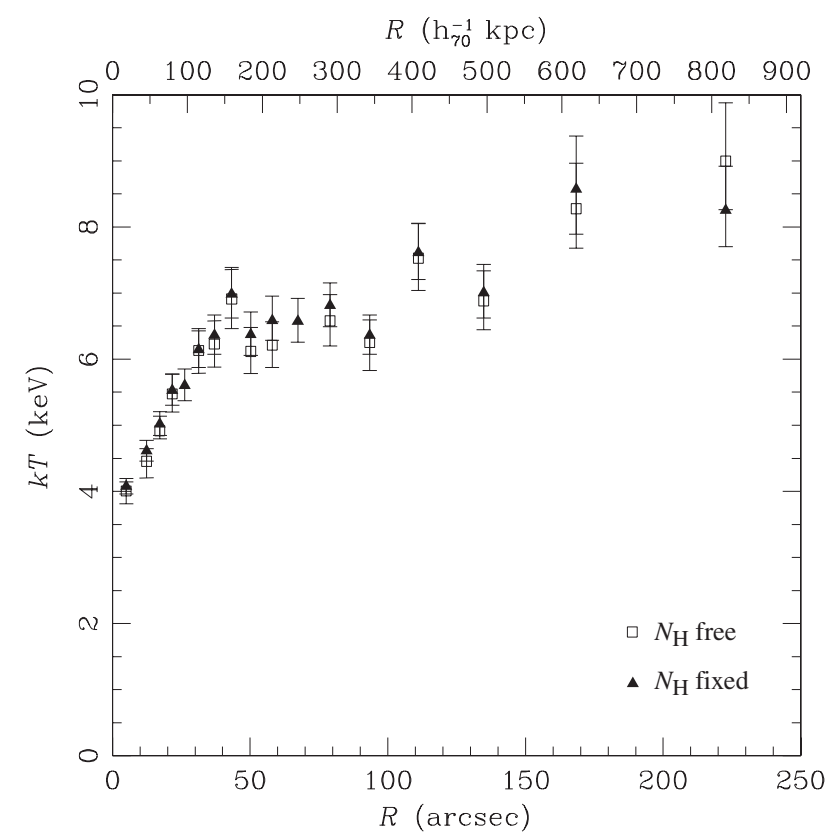

Fig. 11. XMM temperature profile obtained in concentric circles, with the hydrogen absorption column either fixed to the Galactic value (black triangles) or free to vary (empty squares). The temperature is in $\mathrm{keV}$ and the radius in arcsec (bottom axis) or in kpc (top axis).

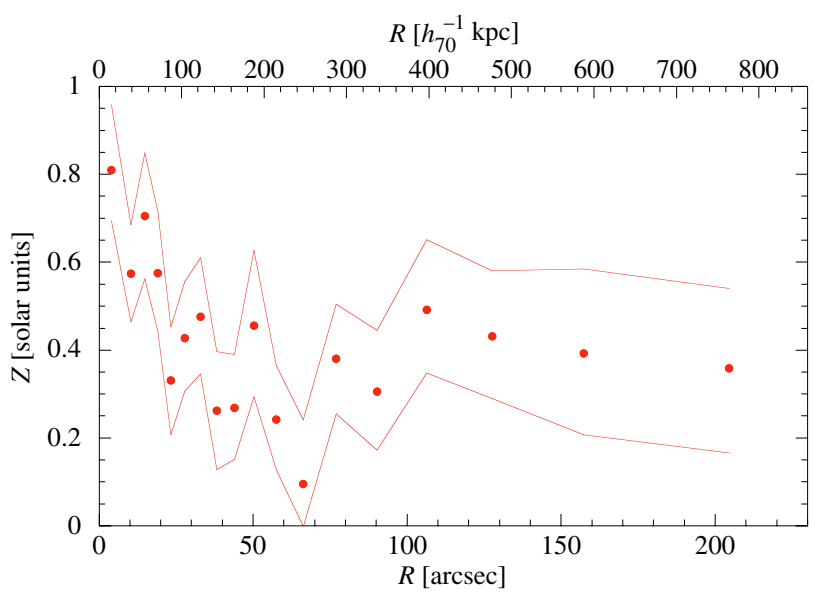

Fig. 12. XMM metallicity profile of the $X$-ray gas in concentric circles, in solar units. Continuous lines correspond to the $90 \%$ confidence level interval (i.e., $2.7 \sigma$ ).

cluster, then remains roughly constant up to a radius of $\sim 350 \mathrm{kpc}$, and increases notably in the regions where the temperature map has indeed revealed the presence of hotter zones (Fig. 10). Note, however, that the error bars on the temperature are large in the outermost bin.

In a similar way, fitting a single MEKAL model, we drew the metallicity profile of the X-ray emitting gas, as shown in Fig. 12. It shows high values between 0.6 and 0.8 (in solar units) in the innermost $100 \mathrm{kpc}$, then varies between 0.2 and 0.5 at larger radii. The mean emission-weighted metallicity within 200 arcsec is about $0.4 Z_{\odot}$, a value usually found in nearby rich clusters (e.g., Fukazawa et al. 2000). The extent to which the hydrogen column density is fixed or not only has a very small effect; therefore, the results related to the metallicity presented below are always obtained by letting $N_{\mathrm{H}}$ remain free.

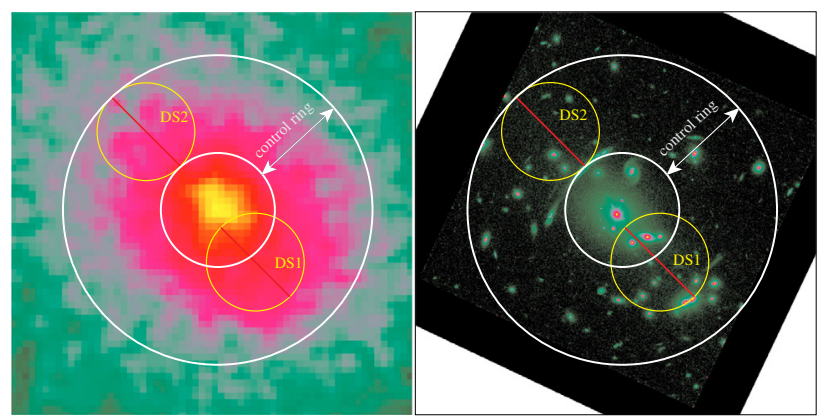

Fig. 13. Spectral extraction regions are shown superimposed on the XMM (left) and HST (right) images. The yellow circles (12 arcsec radius each) show the regions corresponding to the diffuse light sources DS1 and DS2. The inner white circle (14 arcsec radius) is excluded, and the outer white circle ( 38 arcsec radius) binds the ring used as a control.

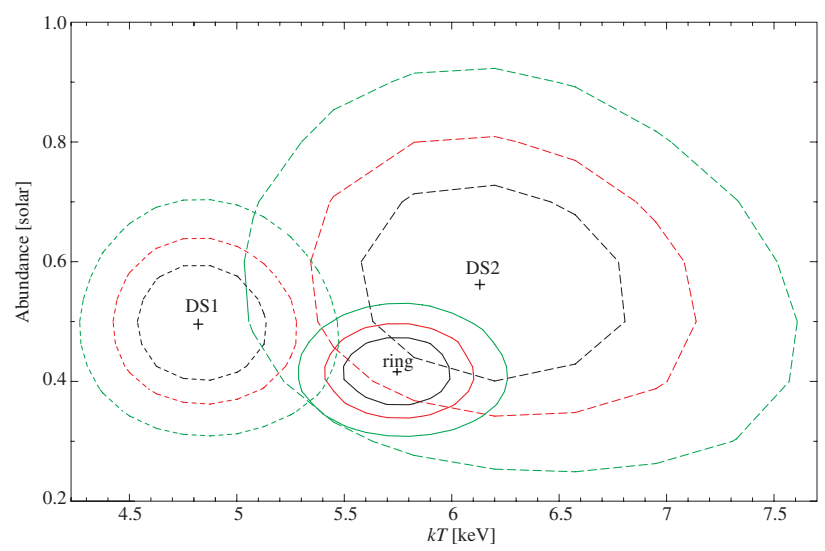

Fig. 14. Confidence levels $(1,2$ and $3 \sigma$, in black, red and green, respectively) for the spectral fits of regions DS1 (short dashes), DS2 (long dashes) and the ring excluding both regions (full lines) (see text for details).

Finally, we obtained the temperature and metallicity at the positions of the two diffuse sources, DS1 and DS2. Figure 13 shows the regions used for spectral extraction, as well as a control ring that excludes the DS2 region and part of the DS1 region.

The temperature and metallicity in the DS1 region are $k T=$ $4.8 \pm 0.3 \mathrm{keV}$ and $Z=0.50_{-0.10}^{+0.11} Z_{\odot}(90 \%$ confidence level $)$, respectively. For the DS2 source we obtain $k T=6.1_{-0.6}^{+0.8} \mathrm{keV}$ and $Z=0.56_{-0.18}^{+0.19} Z_{\odot}$. In order to check if the metallicity in DS2, the region with the higher metallicity and farther from the center, is higher than in its neighborhood, we extract a spectrum in the control ring around the position of the DS2 source, but exclude it and part of the DS1 source (see Fig. 13).

We plotted the confidence level curves for the quantities $k T$ and $Z$ for DS1, DS2, and the control ring in Fig. 14. The ICM temperature and metallicity of the control ring are $k T=$ $5.7 \pm 0.3 \mathrm{keV}$ and $Z=0.42 \pm 0.06 Z_{\odot}$. Although the metallicity of the regions associated with DS1 and DS2 may be higher than in their surroundings, the difference is significant only at $\sim 1 \sigma$. Therefore, a deeper X-ray exposure is required to confirm the differences in metallicities found with the present data.

\section{The building history of Abell 2667}

In this section we summarize the observed properties of the detected diffuse sources in A2667, discuss whether they are to be considered as part of the $\mathrm{cD}$ envelope and describe a possible 
scenario in which their formation is related to the infalling of galaxy groups in the cluster.

\subsection{Diffuse light}

By applying a multi-scale wavelet analysis and reconstruction technique to the HST images of the cluster Abell 2667 in three bands, we have shown the probable existence of one large-scale source of diffuse emission (DS1), in which a faint and more compact extended source (ComDif) is embedded, most likely at the same redshift as the cluster. A second diffuse source may be present (DS2) at a lower significance level.

We measured the optical colors of these sources. The colors of the two sources DS1 and ComDif are close to the color of K-type stars at the cluster redshift (Fig. 6), implying a population of evolved stars. These colors are slightly bluer than those found in a simulated Coma-like cluster at $z=0$ by Sommer-Larsen et al. (2005), probably because of the younger age of the detected diffuse light systems (at least 3.7 Gyrs younger since located at $z=0.233$ ).

VIMOS-IFU spectroscopy reveals a continuum signal at the location of the sources DS1 and ComDif with no apparent emission line brighter than a flux limit $f_{1} \simeq 0.7 \times 10^{-18} \mathrm{erg} \mathrm{s}^{-1} \mathrm{~cm}^{-2}$. The identification of the $\mathrm{Ca} \mathrm{H}$ and $\mathrm{K}$ absorption lines (expected in an evolved stellar population) at the cluster redshift is problematic because of the strongly varying background at the interested spectral position.

The X-ray gas in the DS1 and DS2 regions may be metalricher than their immediate neighborhood (Fig. 14) but this is only a $1 \sigma$ difference. While deeper X-ray data are necessary to confirm these findings, we note that Zaritsky et al. (2002) show that the ICL population can contribute significantly to the metal enrichment of the ICM, via supernova winds, an in situ mechanism that also suggests a spatial correlation between metal richer regions and ICL sources.

Recent numerical simulations also have not yet addressed the question of a possible spatial correlation between the ICM metallicity and the presence of ICL sources. Globally, the metallicity profile in Abell 2667 (see Fig. 13) agrees well with simulations by Romeo et al. (2006). The values close to half solar are also in good agreement with Romeo et al. (2006).

The quantity of matter included in the two detected diffuse light sources remains modest compared to the total cluster mass. While the large-scale diffuse light contribution to the Coma cluster was close to the luminosity of a $\mathrm{cD}$ galaxy (Adami et al. 2005), here this diffuse light only represents a small fraction (about 10-15\%) of the luminosity of the main galaxy, which is $I_{814}=17.2$ (absolute magnitude -23.1). This agrees well with the work of Lin \& Mohr (2004) showing that the ICL contribution increases with cluster mass. We note that we have detected only the sources of diffuse light larger than $\sim 2$ arcsec with a surface brightness brighter than $\sim 26$ in the $F 814 \mathrm{~W}$ filter. Therefore, our total light fraction estimate is only valid for large-scale sources.

The total contribution of these diffuse light sources is equivalent to an $I_{814}=19.4$ galaxy (absolute magnitude -20.9). If we assume that diffuse light is proportional to the infall activity onto the cluster (as suggested by Willman et al. 2004), Abell 2667 is, therefore, apparently a more isolated cluster than the Coma cluster in the cosmic web. However, the link between infalling groups and diffuse light sources is far from clear, and this has to be confirmed by a more specific study of the environment of these two clusters.
Finally, we note that this fraction agreees with the findings of Zibetti et al. (2005), who summed the faint signal from a large sample of rich clusters from the SDSS at roughly the same cosmic epoch, and with the numerical simulations by Willman et al. (2004), who followed the time evolution of the ICL fraction in a Coma-like cluster.

\subsection{Diffuse light sources as part of the brightest cluster galaxy envelope?}

In this work we have made no strict distinction between the brightest cluster galaxy envelope and the diffuse sources found by using the multiscale approach. Indeed, while in numerical simulations it is possible to separate the two stellar populations on dynamical grounds, this is not possible using photometric data. Kelson et al. (2002) used deep long-slit spectroscopic data to show that the outskirts of the brightest cluster galaxy halo in the galaxy cluster Abell 2199 share the same velocity dispersion as the cluster galaxies, with a monotonic increase from a few kpc from the brightest cluster galaxy center outwards. This behavior thus supports a view in which ICL sources and the brightest cluster galaxy envelope originate from the same physical processes.

The detected diffuse sources could, however, be considered as part of the brightest cluster galaxy halo in terms of the usual photometric definition. In other words, an alternative explanation of the presence of sources of diffuse light would be that these are not independent from the dominant galaxy envelope. If we fit elliptical isophotes on the $F 814 W$ HST image after excluding the other visible galaxies using the ellipse package in STSDAS, we find that the surface brightness profile starting from the dominant galaxy center approximately follows an $r^{1 / 4}$ law with an ellipticity growing from $\sim 0.2$ close to the center, to $\sim 0.6,30$ arcsec from the center. In this picture, the sources DS1 and DS2 do not appear as major residuals over the $r^{1 / 4}$ law. This means that the sum of the possible diffuse light sources and of the dominant galaxy contribution is correctly modeled by an $r^{1 / 4}$ law, as long as we allow the ellipticity to grow regularly from the dominant galaxy center to the image borders.

It is difficult, however, to constrain the true ellipticity of the dominant galaxy unambiguously, i.e. the ellipticity corresponding to this galaxy and not due to the addition of independent diffuse light sources. If the true ellipticity of the dominant galaxy really increases radially up to a value of 0.6 far from the center, then the detected diffuse sources, even if they are real, can simply be the external parts of the strongly elliptical central galaxy. If the true ellipticity of the main galaxy remains constant, then the diffuse light sources are independent entities.

Note, however, that up to a certain point these two hypotheses are similar, since it is difficult to estimate a sharp boundary between the brightest cluster galaxy envelope and the nearby diffuse sources. Accretions are probably occurring along the main cluster axis, which is also that of the main central galaxy, so that considering the diffuse sources as independent entities that will soon be accreted by the central galaxy in its external parts along the infalling direction, or considering that the diffuse sources have already been accreted on to the external parts of this galaxy (see, e.g., Richstone 1976) is not very different. A possible way to discriminate between these two explanations would be to get sufficient $\mathrm{S} / \mathrm{N}$ spectra of the diffuse sources to compute their precise redshifts; unfortunately, our spectroscopic data are not deep enough for this purpose.

Another caveat is that elongated $r^{1 / 4}$ halos are normally seen around ellipticals and they are in virial equilibrium (see e.g. 
Arnaboldi et al. 2004; Mihos et al. 2005), so the detected extensions could not be related to the direction of infalling material at all. However, the good correlation between the brightest galaxy halo extension and the infalling directions still suggests a relation between these two components.

\subsection{Infalling directions}

A natural explanation would be that the diffuse sources are concentrations of tidal debris and harassed matter expelled from galaxies by tidal stripping, close galaxy interactions or strong tidal forces close to the cluster center. However, there is only one clear distorted object (ComDif). The remainder of the diffuse sources appear quite smooth. This hypothesis remains to be tested with deeper images but these sources could be the result of already well-mixed old tidal tails. These processes are expected to act on infalling galaxies, and it would not be surprising to find diffuse light sources along the main infall directions, although this view still needs support from more observations and numerical simulations.

The X-ray analysis shows somewhat hotter regions SW and NE of the cluster, which could be due to shock fronts along these two directions produced by infalling material still distant from the cluster center. The error bars on the X-ray gas temperature in the outer zones are, however, large and this scenario has to be considered with caution even if it agreed with the general elongation of the X-ray gas emission (see Fig. 9) and with the hypothesis of matter infalling along a NE to SW direction. This direction matches that joining DS1 and DS2. Moreover, the dynamical analysis we performed using the SG method shows three independent groups (G1, G2, and G3) again defining the same direction. We suggest that G2 and G3 are infalling groups from the surrounding cosmic web.

In order to find evidence for this cosmic web, we used the NASA Extragalactic Database (NED) to search for known galaxy clusters in a $\sim 40 \mathrm{Mpc}$ radius area around Abell 2667 and with redshift between 0.21 and 0.26 . Only two rich galaxy clusters were found, Abell 4041 and Abell S1151 located about $40 \mathrm{Mpc}$ from Abell 2667. These two clusters are located roughly in the same direction as defined by the groups G2-G1 (see Fig. 3). We note, however, that the known cluster population at these redshifts is far from complete, and we cannot conclude that G2 is infalling onto the Abell 2667 cluster core coming from the Abell 4041/AbellS 1151 complex, which is noticeably farther away.

We also searched for all known individual galaxy redshifts between $z=0.21$ and 0.26 in a larger area around Abell 2667. A hundred redshifts are available from the NED database (mainly from the $2 \mathrm{dF}$ survey) and are concentrated in a strip extending from $\delta=-28^{\circ}$ to $\delta=-26^{\circ}$. Applying the SG algorithm to these data, we detected several bound structures, but only one with a binding energy larger than that of G4 (on the order of that of G1) and sampled with more than 10 redshifts. This structure is a group (hereafter G5) located at $\alpha=356.84^{\circ}$ and $\delta=-27.66^{\circ}$ at $\simeq 6$ arcmin from the Abell 2667 center. Its redshift $0.221 \pm$ 0.001 , and its velocity dispersion $164 \mathrm{~km} \mathrm{~s}^{-1}$, corresponding to a mass of $8 \times 10^{12} M_{\odot}$. This structure is located in the same direction as defined by groups G2-G1 (see Fig. 3) and is located at about $26 \mathrm{Mpc}$ from Abell 2667.

The detections of G2, G5, Abell S1151 and Abell 4041 along a direction defined by the elongation of the X-ray emission and by the zones where the X-ray gas is hotter, are all consistent with the hypothesis of a filament crossing Abell 2667 and along which galaxies are flowing onto the cluster; but of course in view of the limited $\mathrm{S} / \mathrm{N}$ of all our data, this scenario remains speculative.

\subsection{G2 infalling characteristics}

G2 is perhaps directly responsible for the formation of the ICL source DS1. If this group is actually infalling onto the core of Abell 2667, we may expect that the low-mass galaxies of this group will be in advance along the infalling direction (see, e.g., Sarazin 1986). As low-mass galaxies are easier to disrupt than massive ones, they could have been destroyed before reaching the Abell 2667 cluster core, therefore contributing to forming the detected diffuse sources.

That G2 is constituted of two relatively bright galaxies (the fourth and seventh brightest galaxies of the cluster, Covone et al. 2006a) suggests that G2 has already started to dissolve in the Abell 2667 potential, as such bright galaxies are expected to show an associated, surrounding faint galaxy group. It is difficult to estimate the original mass of this group, because the absence of any extended X-ray emission (see Fig. 9) acts in favor of a minor group. Recent numerical simulations by Tormen et al. (2004) show that an infalling group with a relative mass compared to the main cluster between 1 and $10 \%$ will keep its intra-group gas during less than 2 Gyrs, but will survive as an independent entity for a longer time (about half of its dark matter staying clustered during up to 3 Gyrs). In this scenario, if G2 has already lost its gas but still exists as an independent pair, this implies that it has been falling onto Abell 2667 for a time between 2 and 3 Gyrs. Assuming that the tidal gravitational forces from Abell 2667 start to be effective in removing the group gas at roughly the virial radius, the corresponding infall velocity of $\mathrm{G} 2$ would then be between 1000 and $1500 \mathrm{~km} \mathrm{~s}^{-1}$.

\section{Summary}

We have proposed a possible scenario for the building process in the core of the galaxy cluster Abell 2667 based on the analyses of optical HST imaging, Chandra and XMM X-ray data, and VIMOS-IFU spectroscopy. Here we summarize our main results:

(i) By means of an iterative multi-scale wavelet analysis and reconstruction of HST images of Abell 2667 in three filters, we have shown the presence of a zone of diffuse emission south west of the cluster center (DS1); a second very faint object, the "compact diffuse source", is observed within DS1. These two sources have similar colors, close to those of old, K-type stars at the cluster redshift. Another diffuse source (DS2) is also possibly detected NE of the center in the reddest filter, but with lower $\mathrm{S} / \mathrm{N}$.

(ii) Four dynamical groups are detected within the cluster core using the Serna \& Gerbal (1996) hierachical method (G1, G2, G3 and G4). One additional group (G5) is identified much farther away $\simeq 6^{\prime}(1.34 \mathrm{Mpc})$ from the cluster center.

(iii) A spectroscopic continuum is detected for DS1 and ComDif, though the strongly spatially varying background and the low $\mathrm{S} / \mathrm{N}$ prevent an unambiguous identification of the expected absorption lines and only allows putting upper values regarding the presence or absence of emission lines.

(iv) The analysis of XMM-Newton and Chandra observations shows that the X-ray emission is elongated along the same direction as defined by DS1, the cluster center, and DS2. The dynamical groups G1, G2, G3, G5, and two rich 
galaxy clusters at $\simeq 40 \mathrm{Mpc}$ (Abell 4041, Abell S1151) from Abell 2667 also define a very similar direction.

(v) The X-ray temperature map shows the presence of a cool core, i.e. a broad cool zone stretching roughly from north to south, which possibly suggests hotter regions towards the NE, SW and NW, though error bars in these zones are large. The gas heating in these hotter regions could be due to shock fronts produced along these directions by infalling material. The general elongation observed agrees with the proposed scenario in which diffuse sources are probably concentrations of tidal debris and harassed matter expelled from infalling galaxies by tidal stripping, so it is expected to be found along the main infall directions. However, this scenario is still speculative at this stage and would require numerical simulations that are beyond the scope of this paper.

Acknowledgements. The authors thank Tatiana Ferraz Laganá for deriving the X-ray temperature profile, Francesco La Barbera for help on stellar photometry, Nicola Napolitano for stimulating discussions, and the referee for constructive remarks that helped to improve the presentation of the results. The authors also acknowledge useful discussions with Geneviève Soucail. G.C. acknowledges financial support from the EURO-3D Research Training Network (funded by the European Commission under the contract No. HPRN-CT-2002-00305), C.A. and F.D. acknowledge support from the PNC, F.D., and G.B.L.N. acknowledge financial support from the CAPES/COFECUB, and JPK from the CNRS and Caltech. This research made use of the NASA/IPAC Extragalactic Database, which is operated by the Jet Propulsion Laboratory, California Institute of Technology, under contract with the National Aeronautics and Space Administration.

\section{References}

Adami, C., Slezak, E., Durret, F., et al. 2005, A\&A, 429, 39

Arnaboldi, M. 2004, in Baryons in Dark Matter Halos, Novigrad, Croatia

Arnaboldi, M., Ortwin, G., Aguerri, J. A. L., et al. 2004, ApJ, 614, L33

Calcáneo-Roldán, C., Moore, B., Bland-Hawthorn, J., Malin, D., \& Sadler, E. M. 2000, MNRAS, 314, 324

Covone, G. 2004, in SF2A-2004: Semaine de l'Astrophysique Française, ed. F. Combes, et al. (EDP Sciences), 521

Covone, G., Kneib, J.-P., Soucail, G., et al. 2006a, A\&A, 456, 409

Covone, G., Kneib, J.-P., Soucail, G., et al. 2006b, in Sciences Perspectives for 3D Spectroscopy. ESO Astrophysics Symposia, ed. M. Kissler-Patig, M. M. Roth, \& J. R. Walsh [arXiv: astro-ph/0601387]
Da Rocha, C., \& Mendes de Oliveira, C. 2005, MNRAS 364, 1069 Dickey, J. M., \& Lockman, F. J. 1990, ARA\&A, 28, 215

Durret, F., Lima, Neto, G. B., \& Forman, W. 2005, A\&A, 432, 809

Feldmeier, J. J., Mihos, J. C., Morrison, H. L., Rodney, S. A., \& Harding, P. 2002, ApJ, 575, 779

Feldmeier, J. J., Mihos, J. C., Morrison, H. L., Harding, P., \& Kaib, N. 2003, Carnegie Observatories Astrophysics Series Vol. 3, Proc. Clusters of galaxies: probes of cosmological structure and galaxy evolution, ed. J. S. Mulchaey, A. Dressler, \& A. Oemler [arXiv: astro-ph/0303340]

Fukazawa, Y., Makishima, K., Tamura, T., et al. 2000, MNRAS, 313, 21 Gonzalez, A. H., Zabludoff, A. I., \& Zaritsky, D. 2005, ApJ, 618, 195

Gregg, M. D., \& West, M. J. 1998, Nature, 396, 549

Gunn, J. E., \& Gott, J. R. III 1972, ApJ, 176, 1

Kaastra, J. S., \& Mewe, R. 1993, A\&AS, 97, 443

Kelson, D. D., Zabludoff, A. I., Williams, K. A., et al. 2002, ApJ, 576, 720

Kinney, A. L., Calzetti, D., Bohlin, R. C., et al. 1996, ApJ, 467, 38

Krick, J. E., Bernstein, R. A., \& Pimbblet, K. A. 2006, AJ, 131, 168

Larson, R. B., Tinsley, B. M., \& Caldwell, C. N. 1980, ApJ, 237, 692

Liedahl, D. A., Osterheld, A. L., \& Goldstein, W. H. 1995, ApJ, 438, L115

Lin, J., \& Mohr, J. J. 2004, ApJ, 617, 879

Łokas, E. L., \& Mamon, G. A. 2003, MNRAS, 343, 401

Lumb, D. H., Warwick, R. S., Page, M., \& De Luca, A. 2002, A\&A, 389, 93

Mihos, J. C. 2003, Carnegie Observatories Astrophysics Series Vol. 3, Proc. Clusters of galaxies: probes of cosmological structure and galaxy evolution, ed. J. S. Mulchaey, A. Dressler, \& A. Oemler [arXiv: astro-ph/0305512] Mihos, J. C., Harding, P., Feldmeier, J., \& Morrison, H. 2005, ApJ, 631, L41 Moore, B., Lake, G., \& Katz, N. 1998, ApJ, 495, 139

Murante, G., Arnaboldi, M., Gerhard, O., et al. 2004, ApJ, 607, L83

Napolitano, N. R., Pannella, M., Arnaboldi, M., et al. 2003, ApJ, 594, 172

Natarajan, P., Kneib, J.-P., \& Smail, I. 2002, ApJ, 580, L11

Pickles, A. J. 1998, PASP, 110, 863

Piffaretti, R., Jetzer, Ph., Kaastra, J. S., \& Tamura, T. 2005, A\&A, 433, 101 Richstone, D. O. 1976, ApJ, 204, 642

Rizza, E., Burns, J. O., Ledlow, M. J., et al. 1998, MNRAS, 301, 328

Romeo, A. D., Sommer-Larsen, J., Portinari, G., \& Antonuccio-Delogu, V. 2006, MNRAS, in press [arXiv: astro-ph/0509504]

Sarazin, C. L. 1986, Rev. Mod. Phys., 58, 1

Serna, A., \& Gerbal, D. 1996, A\&A, 309, 65

Sommer-Larsen, J., Romeo, A. D., \& Portinari, L. 2005, MNRAS, 357, 478

Tormen, G., Moscardini, L., \& Yoshida, N. 2004, MNRAS, 350, 1397

Treu, T., Ellis, R. S., Kneib, J.-P., et al. 2003, 591, 53

Uson, J. M., et al. 1991, ApJ, 369, 46

Willman, B., Governato, F., Wadsley, J., \& Quinn, T. 2004, MNRAS, 355, 159

Zanichelli, A., Garilli, B., Scodeggio, M., et al. 2005, PASP, 117, 1271

Zaritsky, D., Gonzalez, A. H., \& Zabludoff, A. I. 2004, ApJ, 613, L93

Zibetti, S., White, S. D. M., Schneider, D. P., \& Brinkmann, J. 2005, MNRAS, 358,949 